\title{
Diagnostic accuracy of Onen's Alternative Grading System combined with Doppler evaluation of ureteral jets as an alternative in the diagnosis of obstructive hydronephrosis in children
}

\author{
Jose Bessa Junior ${ }^{\text {Corresp., }}{ }^{1}$, Cicilia M Rodrigues ${ }^{1}{ }^{\text {, }}$ Maria Cristina Chammas ${ }^{2}$, Eduardo P Miranda ${ }^{3}$, Cristiano M \\ Gomes ${ }^{4}$ ， Paulo R Moscardi ${ }^{4}$, Marcia C Bessa ${ }^{5}$, Carlos A Molina ${ }^{1}$, Ricardo B Tiraboschi ${ }^{1}$, Jose M Netto $^{6}$, \\ Francisco T Denes ${ }^{4}$ \\ ${ }^{1}$ Division of Urology/Public Health, Medical School, Universidade Estadual de Feira de Santana, Feira de Santana, BA, Brazil \\ 2 Department of Radiology, Medical School, Universidade de São Paulo, São Paulo, SP, Brazil \\ 3 Division of Urology, Medical School, Universidade Federal do Ceará, Fortaleza, CE, Brazil \\ 4 Division of Urology, Medical School, Universidade de São Paulo, São Paulo, SP, Brazil \\ 5 Division of Pediatrics, Medical School, Universidade Estadual de Feira de Santana, Feira de Santana, BA, Brazil \\ 6 Division of Urology, Hospital e Maternidade Therezinha de Jesus of the School of Medical Science and Health of Juiz de Fora (HMTJ-SUPREMA), \\ Universidade Federal de Juiz de Fora, Juiz de Fora, MG, Brazil \\ Corresponding Author: Jose Bessa Junior \\ Email address: bessa@uefs.br
}

Introduction: Ureteropelvic junction obstruction (UPJO) is a common congenital anomaly leading to varying degrees of hydronephrosis $(\mathrm{HN})$, ranging from no apparent effect on the renal function to atrophy. Evaluation of these children is based on Diuretic Renal Scintigraphy (DRS) and Ultrasonography (US). Recent studies have suggested that new parameters of conventional and color Doppler ultrasonography (CDUS) may be useful in discriminating which kidneys are obstructed. The present study aims to assess the diagnostic accuracy of such parameters in the diagnosis of obstruction in children with UPJO. Methods: We evaluated 44 patients (33 boys) with a mean age of $6.53 \pm 4.39$ years diagnosed with unilateral high-grade hydronephrosis (SFU grades 3 and 4). All underwent DRS and index tests (conventional US and CDUS to evaluate ureteral jets frequency) within a maximum interval of 2 weeks. Hydronephrotic units were reclassified according to the alternative grading system (AGS) proposed by Onen et al. Obstruction in the DRS was defined as a differential renal function $<40 \%$ on the affected side and/or features indicating poor drainage function like $\mathrm{T} 1 / 2>20$ minutes after the administration of furosemide, and a plateau or ascending pattern of the excretion curve. Results: Nineteen hydronephrotic units (43.1\%) were obstructed. Some degree of cortical atrophy - grades 3 (segmental) or 4 (diffuse) - was present in those obstructed units. AGS grades had $100 \%$ sensitivity, $76 \%$ of specificity and $86.4 \%$ of accuracy. The absence of ureteral jets had a sensitivity of $73.68 \%$, a specificity of $100 \%$ with an accuracy of $88.6 \%$. When we analyzed 
the two aspects together and considered obstructed the renal units classified as AGS grade 3 or 4 with no jets, sensitivity increased to $78.9 \%$, accuracy to $92 \%$, remaining with a maximum specificity of $100 \%$. These features combined would allow us to avoid performing DRS in $61 \%$ of our patients, leaving more invasive tests to inconclusive cases. Conclusions:Although DRS remains the mainstay to distinguishing obstructive from nonobstructive kidneys, grade of hydronephrosis and frequency of ureteral jets, independently or in combination may be a reliable alternative in the mostly cases. This alternative approach has high accuracy, it is less invasive, easily reproducible and may play a role in the diagnosis of obstruction in pediatric population. 


\section{Diagnostic accuracy of Onen's Alternative Grading System}

\section{2 combined with Doppler evaluation of ureteral jets as an}

3 alternative in the diagnosis of obstructive hydronephrosis in

4 children.

5 Jose de Bessa Junior ${ }^{1}$, Cicilia M Rodrigues ${ }^{1}$, Maria C Chammas ${ }^{2}$, Eduardo P Miranda ${ }^{3}$, Cristiano M

6 Gomes $^{4}$, Paulo R M Moscardi ${ }^{4}$, Marcia C Bessa ${ }^{5}$, Carlos A Molina ${ }^{1}$, Ricardo B Tiraboschi ${ }^{1}$, Jose Murillo

7 Netto $^{6}$, Francisco T Denes ${ }^{4}$

$8{ }^{1}$ Division of Urology/Public Health, Medical School, Universidade Estadual de Feira de Santana, Feira de

9 Santana, BA, Brazil

2 Department of Radiology, Medical School, Universidade de São Paulo, São Paulo, SP, Brazil

${ }^{3}$ Division of Urology, Medical School, Universidade Federal do Ceará, Fortaleza, CE, Brazil

${ }^{4}$ Division of Urology, Medical School, Universidade de São Paulo, São Paulo, SP, Brazil

${ }^{5}$ Division of Pediatrics, Medical School, Universidade Estadual de Feira de Santana, Feira de Santana, BA, Brazil

${ }^{6}$ Division of Urology,Hospital e Maternidade Therezinha de Jesus of the School of Medical Science and

Corresponding Author:

Jose de Bessa Junior ${ }^{1}$

Av Getulio Vargas, 744, Feira de Santana, BA, Brazil 
24

25

26

27

28

29

30

31

32

33

34

\section{Abstract}

Introduction: Ureteropelvic junction obstruction (UPJO) is a common congenital anomaly leading to varying degrees of hydronephrosis $(\mathrm{HN})$, ranging from no apparent effect on the renal function to atrophy. Evaluation of these children is based on Diuretic Renal Scintigraphy (DRS) and Ultrasonography (US). Recent studies have suggested that new parameters of conventional and color Doppler ultrasonography (CDUS) may be useful in discriminating which kidneys are obstructed. The present study aims to assess the diagnostic accuracy of such parameters in the diagnosis of obstruction in children with UPJO.

Methods: We evaluated 44 patients (33 boys) with a mean age of $6.53 \pm 4.39$ years diagnosed with unilateral high-grade hydronephrosis (SFU grades 3 and 4). All underwent DRS and index tests (conventional US and CDUS to evaluate ureteral jets frequency) within a maximum interval of 2 weeks. Hydronephrotic units were reclassified according to the alternative grading system (AGS) proposed by Onen et al. Obstruction in the DRS was defined as a differential renal function $<40 \%$ on the affected side and/or features indicating poor drainage function like T1/2 $>20$ minutes after the administration of furosemide, and a plateau or ascending pattern of the excretion curve.

Results: Nineteen hydronephrotic units $(43.1 \%)$ were obstructed. Some degree of cortical atrophy - grades 3 (segmental) or 4 (diffuse) - was present in those obstructed units. AGS grades had $100 \%$ sensitivity, $76 \%$ of specificity and $86.4 \%$ of accuracy. The absence of ureteral jets had a sensitivity of $73.68 \%$, a specificity of $100 \%$ with an accuracy of $88.6 \%$. When we analyzed the two aspects together and considered obstructed the renal units classified as AGS grade 3 or 4 with no jets, sensitivity increased to $78.9 \%$, accuracy to $92 \%$, remaining with a maximum 
47 specificity of $100 \%$. These features combined would allow us to avoid performing DRS in $61 \%$

48 of our patients, leaving more invasive tests to inconclusive cases.

49 Conclusions: Although DRS remains the mainstay to distinguishing obstructive from non-

50 obstructive kidneys, grade of hydronephrosis and frequency of ureteral jets, independently or in

51 combination may be a reliable alternative in the most cases. This alternative approach has high

52 accuracy, it is less invasive, easily reproducible and may play a role in the diagnosis of

53 obstruction in pediatric population 
56

57

58

59

60

61

62

63

64

65

66

67

68

\section{.INTRODUCTION}

56

57

Antenatal hydronephrosis $(\mathrm{HN})$ is a common condition seen in 1 to $5 \%$ of all pregnancies. Despite this high incidence seen, only a small percentage of cases will persist and represent a pathology in the postnatal period (Lee et al. 2006). The most common clinically etiology of hydronephrosis is ureteropelvic junction obstruction (UPJO), accounting for more than $50 \%$ of cases of high-grade $\mathrm{AHN}$ and represents an important cause of kidney damage in infants (Lee et al. 2006; Vemulakonda et al. 2014). However, it often develops with no apparent clinical significance as it may not lead to renal parenchyma injury and may ultimately undergo spontaneous resolution in most children (Eskild-Jensen et al. 2005; Heinlen et al. 2009; Ozcan et al. 2004).

Most cases of HN are diagnosed in the antenatal period, having relatively well defined protocols for evaluation and follow-up (Eskild-Jensen et al. 2005; Lee et al. 2006; Ozcan et al. 2004; Ransley et al. 1990). Delayed diagnosis of obstructed HN occurs most often in those who lost follow-up or had a progressive worsening of a lower-grade HN. The best way to evaluate and follow-up these older patients, however, is yet to be established. Most protocols recommend serial monitoring with ultrasound (US) and radionucleide diuretic renal scintigraphy (DRS), to determine the degree of $\mathrm{HN}$ and parenchyma damage as well the differential renal function (DRF) (Dhillon 1998; Gatti et al. 2001; Hafez et al. 2002; Lupton \& Testa 1992; Onen 2007; Peters 1995). Children with high-grade $\mathrm{HN}$ have a higher risk of progression, and kidney damage (Lee et al. 2006). Surgery is indicated upon worsening of hydronephrosis, lithiasis, recurrent infections or when renal function deteriorates (Csaicsich et al. 2004; Gatti et al. 2001; Hafez et al. 2002; Onen et al. 2002). 
79

80

be regularly repeated, which might have some harmful consequences in children. Therefore, many studies have investigated extended uses of US in order to minimize the necessity of a DRS. It has been postulated that semi-automated quantitative analysis of US images in HN kidneys may have a potential clinical utility, reducing up to $62 \%$ of DRS (Cerrolaza et al. 2016). Other studies have shown good correlation between sonographic findings such as grade of hydronephrosis and ureteral jets pattern and DRF, suggesting a possible role for such parameters in the long-term monitoring of these patients (de Bessa et al. 2008; Erickson et al. 2007; Shapiro et al. 2008; Tekgul et al. 2012; Walker et al. 2015).

The possibility of using an easily available, non-invasive and low-cost method such as ultrasound as an alternative to DRS is certainly attractive. This could be accomplished with an objective assessment of functional and morphological US parameters. The aim of the present study is to evaluate the diagnostic accuracy of renal US and ureteral jet frequency using Doppler US in the bladder for the diagnosis of obstruction in children older than two years old with high-grade unilateral $\mathrm{HN}$.

\section{MATERIALS AND METHODS}

This study was designed based on the most stringent methodological recommendations of the guideline Standard for Reporting of Diagnostic Accuracy (Bossuyt et al. 2003). Eligible patients were 2 or more years of age and had hydronephrosis grade 3 or 4 according to the Society for Fetal Urology (SFU) grading system (Fernbach et al. 1993). Grades 3 and 4 are defined by SFU as dilatation of the renal pelvis, and all calyces visible with normal renal parenchyma (grade 3) or atrophic (grade 4). Children with a solitary kidney, bilateral 
102 hydronephrosis, neurogenic bladder, hydroureteronephrosis and vesicoureteral reflux were 103 excluded. number 166/04). Patients agreed to participate after full disclosure of its purposes, and written

106

107

108 consent was obtained from all participants or their legal representatives.

Data were collected between October 2006 and June 2014. All patients underwent kidney US, color Doppler ultrasonography to evaluate ureteral jets frequency (UJF) - Index Test - as well as DRS. All tests were performed within a maximum interval of 2 weeks by independent examiners, experienced in clinical research, who were blinded to the results of the other method. The reports and images were reviewed and cases were reclassified according to the alternative grading system classification (AGS) proposed by Onen et al. (Onen 2007) - Figure 1. We used previously described criteria for correlation between the two systems with SFU-1 and SFU-2 hydronephrosis considered as AGS-1 since both present renal pelvic dilatations alone or associated with minimal calyceal dilatation. SFU-3 with calyceal dilation was considered as AGS-2 and SFU-4 classified as either AGS-3 (when renal parenchymal thickness represented half or less of the contralateral normal kidney) or AGS-4 if there was severe parenchymal loss (cyst-like kidney) (Onen 2007).

\section{Ureteral Jets on Bladder Ultrasound}

The technique for evaluation of ureteral jets has been previously described (de Bessa et al. 2008). Subjects were evaluated in dorsal-horizontal decubitus. Employing color Doppler mode, the bladder was examined in the transverse section at the level of the trigone to observe both ureteral orifices simultaneously. The jets were counted during five minutes with the 
125 ureteral jets frequency (UFJ) on the hydronephrotic unit side as well as the normal side considered

126 for the subsequent analysis. Relative jet frequency (RJF) was established and defined as the ratio

127 between the UJF in the affected side and the sum of the frequency of jets observed bilaterally in

128 the same period. We considered obstructed, kidneys in which no jets were observed in the

129 hydronephrotic side (UFJ $=0)$.

130

131 Diuretic Renal Scintigraphy

DRS was performed with $99 \mathrm{mTc}-\mathrm{DTPA}$ at a dose of 70 to $120 \mathrm{MBq}$ according to the body

surface area of the child. Patients were positioned supine and received intravenous hydration (10$15 \mathrm{ml} / \mathrm{Kg}$ saline) before the injection of the radiotracer.

Image acquisition began immediately after administration of the radiotracer. In the

early stage of flow, for 1 minute, images were obtained every 2 seconds. Between the first and third minutes, one image was obtained every 5 seconds, and after that the dynamic sequence consisted of one image per minute until the end of the exam. The DRF, expressed as the percentage of each kidney function about overall renal function was calculated according to the relative drug uptake at the initial phase. required to excrete half of the renal pelvis radiotracer measured at the moment of furosemide 144 injection.

We considered obstruction when there were signs of kidney damage including impaired differential renal function $<40 \%$ and/or features indicating poor drainage function like

$147 \mathrm{~T} 1 / 2>20$ minutes after the administration of furosemide, and a plateau or ascending pattern of the 
148 excretion curve (Fernbach et al. 1993).

149

150

151

152

153

154

155

156

157

158

159

160

161

162

163

164

165

166

167

168

169

\section{Statistical Analysis}

Measurement of sensitivity, specificity, accuracy, positive and negative predictive values, as well as positive and negative Likelihood ratios are described with their respective confidence intervals of $95 \%$. Kruskal-Wallis test was used to compare DRF between groups. All tests were two-sided and $\mathrm{p}$ values less than $0.05(\mathrm{p}<0.05)$ were considered statistically significant. The analyses were performed using a statistical software (GraphPad Prism, version 7.0.3, GraphPad Software, San Diego, CA, USA).

\section{RESULTS}

Of 46 patients who were eligible for the study, two (4.3\%) refused to participate. Forty-four subjects were included with a mean age of $6.5 \pm 4.4$ years (range 2 to 17 ) and a median age of 4.9 years. Of the patients, 33 were boys $(75.0 \%)$ and 11 were girls $(25.0 \%)$,. The left kidney was affected in $29(65.9 \%)$ children and the right in $15(34.1 \%)$.

Based on the US and UJF (index test), 25 (56.8\%) kidneys were classified as AGS-3 or AGS-4, of these, $14(56.0 \%)$ had no detectable ureteral jets. DRS evaluation (reference test) in the same group of 25 children was consistent with significant obstruction associated DRF impairment (DRF $<40 \%$ ) in $19(76.0 \%)$ kidneys, including 8 with AGS-4 and 11 with AGS-3. No ureteral jets were detected in 8 kidneys with HN AGS-4 and in 6 of the AGS-3 kidneys. Ureteral jets were present in all of the 19 kidneys classified as AGS-2, and none had abnormal excretion curve nor DRF impairment on DR (Figure 2). In other words, 19 (43.2\%) patients had significant 
170 obstruction kidney including 8 AGS-4 and 11 AGS-3, while 25 (56.8\%) were unobstructed,

171 including 19 AGS-2 and 6 AGS-3. (Figure 2).

173 The remaining 17 cases had DRF $>40 \%$ and were considered as non-obstructive $\mathrm{HN}$ as the 174 radiotracer curve of excretion were descendant with $\mathrm{T}^{1} \frac{1}{2}$ less than 20 minutes. preserved renal function. Of the 17 units classified as Grade 3, $11(64.7 \%)$ had renal impairment, and the other $6(35.3 \%)$ had a normal renal function (Figure 3).

Measures of diagnostic accuracy, positive and negative predictive values and overall accuracy of conventional ultrasound and UJF on Doppler US in the diagnosis of obstructive hydronephrosis are described in Table 1.

As DRS is a challenging diagnostic method with possible negative impact in children following repeated testing, there is a search for less invasive and reliable methods capable of identifying significant obstruction in a timely fashion. The cornerstone of a diagnostic test to these patients should be to discriminate cases more likely to require surgical intervention before renal function deteriorating, from those with innocuous HN._The present study has demonstrated that the combination of morphological and functional parameters of US can safely distinguish obstructed kidneys in children older than two years old. 
193 the left side was more affected than right. These findings are consistent with other series in the

194 literature (Fernbach et al. 1993; Onen 2007; Palmer et al. 1998; Peters 1995). We had a higher

195 incidence of high-grade $\mathrm{HN}$ in comparison to newborns. This fact has also been reported by by

196 Sibai et al. (Sibai et al. 2001) who reported a higher proportion of $\mathrm{HN}$ units with $\mathrm{DRF}<40 \%$ in

197 this subset of patients. Apparently, this population is different from most studied groups whose

198 diagnosis is made prenatally. The majority of prenatal $\mathrm{HN}$ resolves in a mean time of 30 months $_{2}$

199 and those that require intervention will usually be operated in mean age of 5 months (Ulman et al. 200 2000).

In the present study, the prevalence of obstructive $\mathrm{HN}$ in children older than two 202 years was $43.6 \%$, which is higher than the reported range of 15 to $25 \%$ (Eskild-Jensen et al. 2005; Palmer et al. 1998). The mean age of the children in this study was 6.53 years old (range 2 to 17 ). This delayed diagnosis might be an effect of asymptomatic individuals who have failed to be identified during prenatal care and incidentally found $\mathrm{HN}$ on the abdominal US done for another reason. classification system for children after their second year of life (Onen 2007; Sibai et al. 2001). In other words, dividing HN SFU grade 4 into AGS grades 3 and 4, may provide valuable information in the follow-up of high-grade hydronephrosis. Also, T1/2 has a limited utility in certain age groups, as it has been demonstrated that this parameter might be abnormal in younger children without major obstruction to urine outflow (Ulman et al. 2000). It is an extremely sensitive test but has a high percentage of false positive, which limits its applicability for defining obstruction. However, 
215 it has tremendous value in ruling out obstruction, specifically when Twhen $\mathrm{T}^{1} / 2<20$ minutes and

216 curves display with good drainage pattern. obstructed had some degree of cortical atrophy (segmental or diffuse). In contrast, all units that had no cortical atrophy and were classified as AGS-2 or SFU-3 were not obstructed and had DRF $>40 \%$. According to our findings, the presence of atrophy (AGS-3 or 4 or SFU-4) is a parameter with $100 \%$ sensitivity, specificity of $76 \%$ and positive probability index (IP+) of 4.2 . Furthermore, the presence of diffuse cortical atrophy (AGS-4) alone had a specificity of $100 \%$, meaning that it

223

224

226

227

228

229

230

231

232 233 consistently predicts kidney damage due to obstruction when present._Similarly ${ }_{2}$ two othersother studies demonstrated that sonographic findings can be useful to predict deterioration in kidney function in prenatally hydronephrosis. Scalabre et al. also demonstrated that abnormal parenchymal thickness was a significant predictor of surgical intervention in newborns with prenatally diagnosed unilateral urinary tract dilation (Scalabre et al. 2017). Moreover, Nguyen et al.(UTD classification system) showed that the anteroposterior diameter of the renal pelvis has similar predictive properties to Onen's classification (Nguyen et al. 2014). Considering parenchymal atrophy, SFU and UTD system are similar, differing from AGS classification that stratifies it in diffuse or focal atrophy, which may be more precise as we demonstrated herein and described above. Patients with diffuse atrophy had a 100\% specificity while those with atrophy not specified (diffuse or focal) had only $76 \%$ specificity regarding obstruction. We believe that both systems add value to the original SFU classification. However, further studies are required to define valid cutoffs for pelvic diameters in children older than two years.

Ultimately, during initial US assessment, we could reliably conclude that kidneys with HN and diffuse cortical atrophy (AGS-4) are obstructed, and those with no atrophy (AGS-2) are nonobstructed. Sequential tests would be then required for AGS-3 patients. Recently, Onen's 
239 hydronephrosis grading system has been updated. Parameters such as cortical parenchyma less

240 than $3 \mathrm{~mm}$, the disappearance of corticomedullary differentiation, the absence of medullary

241 parenchyma, and significant hyperechogenicity also define AGS grade 4, which are by our findings

242 (Onen 2016). Likewise, analysis of UJF has yielded specificity of $100 \%$ and sensitivity of $73 \%$,

243 with an overall accuracy of $88.6 \%$. Thus, the absence of jets confirms that obstruction is present

244 while the presence of any jet does not rule out obstruction, given the possibility of occasionally

245 scattered jets less frequently than the contralateral unobstructed unit. We have previously

246 demonstrated that despite higher specificity found in the absence of ureteral jets (RJF=0), the RJF

247 values that yielded the best accuracy were $<25 \%$ (de Bessa et al. 2008). However, we believe that

248 complete absence of jets in the hydronephrotic side is potentially more important in clinical

249 decision-making as it simplifies the test and practically confirms obstruction. Finally, a

250 combination of both US parameters (morphological and functional), that is AGS-3/4 and UJF=0

251 provided a specificity of $100 \%$ and increased the sensitivity to $78.9 \%$ and accuracy to 90.9 .

252

DRS is still considered the gold standard for the evaluation of HN. It has a

253

254

255

256

257

258

259

260

261

significant role in defining obstruction, confirming indications for surgical treatment and postoperative monitoring. However, the present study allows us to suggest that the association of renal US and analysis UJF may play a role in the diagnosis of obstruction in children $>2$ years of age with HN. It has numerous advantages since it is more accessible, non-invasive, with lower cost, does not involve radiation and provides excellent diagnostic accuracy. These characteristics qualify the use of this method in the initial evaluation of hydronephrosis, adding another tool for identification and screening of obstructed and non-obstructed units. In other words, we could have avoided performing DRS in up to $61 \%$ of our cases, represented by the spectral extremes of the series (AGS-2 with present UJ or AGS-4 with absent UJ). Future prospective and multicenter 
262 studies are necessary to better define the diagnostic role and possible prognostic value of these

263 parameters in the evaluation of hydronephrosis in the neonatal population. Further studies are also

264 necessary to better understand the role of ureteral jets in evaluating post-operative results and need

265 for reintervention.

266

267 CONCLUSIONS

268

269

Although DRS is still the gold standard for the diagnosis of obstructive HN, the association of HN grading and absence of ureteral jets (independently or combined) on color Doppler US is an accurate and easily reproducible option in this scenario. These tests have also demonstrated high sensitivity and specificity, which may help in the diagnosis of obstruction in older children with HN.

\section{ACKNOWLEDGMENTS}

276

of his hydronephrosis grading system in this study. 
280

281

282

283

284

285

286

287

288

289

290

291

292

293

294

295

296

297

298

299

300

301

302

303

304

305

306

307

308

309

310

311

312

313

314

315

316

317

318

319

320

321

322

323

324

325

326

\section{REFERENCES}

Bossuyt PM, Reitsma JB, Bruns DE, Gatsonis CA, Glasziou PP, Irwig LM, Lijmer JG, Moher D, Rennie D, de Vet HC, and Standards for Reporting of Diagnostic A. 2003. Towards complete and accurate reporting of studies of diagnostic accuracy: the STARD initiative. Standards for Reporting of Diagnostic Accuracy. Clin Chem 49:1-6.

Cerrolaza JJ, Peters CA, Martin AD, Myers E, Safdar N, and Linguraru MG. 2016. Quantitative Ultrasound for Measuring Obstructive Severity in Children with Hydronephrosis. J Urol 195:1093-1099. 10.1016/j.juro.2015.10.173

Csaicsich D, Greenbaum LA, and Aufricht C. 2004. Upper urinary tract: when is obstruction obstruction? Curr Opin Urol 14:213-217.

de Bessa J, Jr., Denes FT, Chammas MC, Cerri L, Monteiro ED, Buchpiguel CA, Cerri GG, and Srougi M. 2008. Diagnostic accuracy of color Doppler sonographic study of the ureteric jets in evaluation of hydronephrosis. J Pediatr Urol 4:113-117. 10.1016/j.jpurol.2007.10.013

Dhillon HK. 1998. Prenatally diagnosed hydronephrosis: the Great Ormond Street experience. Br J Urol 81 Suppl 2:39-44.

Erickson BA, Maizels M, Shore RM, Pazona JF, Hagerty JA, Yerkes EB, Chaviano AH, Kaplan WE, and Cheng EY. 2007. Newborn society of fetal urology grade 3 hydronephrosis is equivalent to preserved percentage differential function. J Pediatr Urol 3:382-386. 10.1016/j.jpurol.2007.01.196

Eskild-Jensen A, Gordon I, Piepsz A, and Frokiaer J. 2005. Congenital unilateral hydronephrosis: a review of the impact of diuretic renography on clinical treatment. J Urol 173:1471-1476. 10.1097/01.ju.0000157384.32215.fe

Fernbach SK, Maizels M, and Conway JJ. 1993. Ultrasound grading of hydronephrosis: introduction to the system used by the Society for Fetal Urology. Pediatr Radiol 23:478-480.

Gatti JM, Broecker BH, Scherz HC, Perez-Brayfield MR, and Kirsch AJ. 2001. Antenatal hydronephrosis with postnatal resolution: how long are postnatal studies warranted? Urology 57:1178.

Hafez AT, McLorie G, Bagli D, and Khoury A. 2002. Analysis of trends on serial ultrasound for high grade neonatal hydronephrosis. J Urol 168:1518-1521. 10.1097/01.ju.0000028784.80359.0a

Heinlen JE, Manatt CS, Bright BC, Kropp BP, Campbell JB, and Frimberger D. 2009. Operative versus nonoperative management of ureteropelvic junction obstruction in children. Urology 73:521-525; discussion 525. 10.1016/j.urology.2008.08.512

Lee RS, Cendron M, Kinnamon DD, and Nguyen HT. 2006. Antenatal hydronephrosis as a predictor of postnatal outcome: a meta-analysis. Pediatrics 118:586-593. 10.1542/peds.2006-0120

Lupton EW, and Testa HJ. 1992. The obstructive diuresis renogram: an appraisal of the significance. J Urol 147:981-983.

Nguyen HT, Benson CB, Bromley B, Campbell JB, Chow J, Coleman B, Cooper C, Crino J, Darge K, Herndon $C D$, Odibo AO, Somers MJ, and Stein DR. 2014. Multidisciplinary consensus on the classification of prenatal and postnatal urinary tract dilation (UTD classification system). J Pediatr Urol 10:982998. 10.1016/j.jpurol.2014.10.002

Onen A. 2007. An alternative grading system to refine the criteria for severity of hydronephrosis and optimal treatment guidelines in neonates with primary UPJ-type hydronephrosis. J Pediatr Urol 3:200-205. 10.1016/j.jpurol.2006.08.002

Onen A. 2016. Üreteropelvik bileşke darlığı. Çocuk Cerrahisi Dergisi 30:55-79.

Onen A, Jayanthi VR, and Koff SA. 2002. Long-term followup of prenatally detected severe bilateral newborn hydronephrosis initially managed nonoperatively. J Urol 168:1118-1120. 10.1097/01.ju.0000024449.19337.8d

Ozcan Z, Anderson PJ, and Gordon I. 2004. Prenatally diagnosed unilateral renal pelvic dilatation: a dynamic condition on ultrasound and diuretic renography. J Urol 172:1456-1459. 
Palmer LS, Maizels M, Cartwright PC, Fernbach SK, and Conway JJ. 1998. Surgery versus observation for managing obstructive grade 3 to 4 unilateral hydronephrosis: a report from the Society for Fetal Urology. J Urol 159:222-228.

Peters CA. 1995. Urinary tract obstruction in children. J Urol 154:1874-1883; discussion 1883-1874.

Ransley PG, Dhillon HK, Gordon I, Duffy PG, Dillon MJ, and Barratt TM. 1990. The postnatal management of hydronephrosis diagnosed by prenatal ultrasound. J Urol 144:584-587; discussion 593-584.

Scalabre A, Demede D, Gaillard S, Pracros JP, Mouriquand P, and Mure PY. 2017. Prognostic Value of Ultrasound Grading Systems in Prenatally Diagnosed Unilateral Urinary Tract Dilatation. J Urol 197:1144-1149. 10.1016/j.juro.2016.11.103

Shapiro SR, Wahl EF, Silberstein MJ, and Steinhardt G. 2008. Hydronephrosis index: a new method to track patients with hydronephrosis quantitatively. Urology 72:536-538; discussion 538-539. 10.1016/j.urology.2008.02.007

Sibai H, Salle JL, Houle AM, and Lambert R. 2001. Hydronephrosis with diffuse or segmental cortical thinning: impact on renal function. J Urol 165:2293-2295.

Tekgul S, Riedmiller H, Hoebeke P, Kocvara R, Nijman RJ, Radmayr C, Stein R, Dogan HS, and European Association of U. 2012. EAU guidelines on vesicoureteral reflux in children. Eur Urol 62:534-542. 10.1016/j.eururo.2012.05.059

Ulman I, Jayanthi VR, and Koff SA. 2000. The long-term followup of newborns with severe unilateral hydronephrosis initially treated nonoperatively. J Urol 164:1101-1105.

Vemulakonda V, Yiee J, and Wilcox DT. 2014. Prenatal hydronephrosis: postnatal evaluation and management. Curr Urol Rep 15:430. 10.1007/s11934-014-0430-5

Walker MR, Babikian S, Ernest AJ, Koch TS, Lustik MB, Rooks VJ, and McMann LP. 2015. Sonographic evaluation of hydronephrosis in the pediatric population: is well-tempered sonography necessary? J Ultrasound Med 34:655-662. 10.7863/ultra.34.4.655 


\section{ABBREVIATIONS}

354 99m Tc-DTPA Technetium-99m Diethylene Triamine Pentaacetic Acid

355 AGS Onen's Alternative Grading System

356 DRF Differential Renal Function

357 FUJ Frequency of Ureteral Jets

358 HN hydronephrosis

359 RJF Relative Jet Frequency

360 SFU Society for Fetal Urology

361 UTD Urinary Tract Dilation 


\section{Table 1 (on next page)}

Measurements of diagnostic accuracy.

${ }^{*} p<0.001 ;$ PPV (Positive Predictive Value); NPV (Negative Predictive Value) ,95\% CI(Confidence Interval 95\%). 


\begin{tabular}{|c|c|c|c|c|c|}
\hline & Sensitivity & Specificity & PPV & NPV & Accuracy \\
\hline & $(95 \% \mathrm{Cl})$ & $(95 \% \mathrm{Cl})$ & $(95 \% \mathrm{Cl})$ & $(95 \% \mathrm{Cl})$ & $(95 \% \mathrm{Cl})$ \\
\hline \multirow[t]{2}{*}{ AGS $3 / 4^{*}$} & $100 \%$ & $76 \%$ & $76 \%$ & $100 \%$ & $86.4 \%$ \\
\hline & {$[76-100]$} & [55 - 90.] & {$[55-90]$} & [82-100] & {$[76-96]$} \\
\hline \multirow[t]{2}{*}{ AGS $4^{*}$} & $42 \%$ & $100 \%$ & $100 \%$ & $69.4 \%$ & $75 \%$ \\
\hline & {$[20-66]$.} & [86 - 100] & {$[76-100]$} & {$[51-83]$} & {$[62-87]$} \\
\hline \multirow{2}{*}{$\begin{array}{l}\text { Absence of } \\
\text { Ureteral Jets* }\end{array}$} & $73.6 \%$ & $100 \%$ & $100 \%$ & 83.3 & $88.6 \%$ \\
\hline & [53-93] & [76 - 100] & {$[76-100]$} & {$[65-94]$} & [79 - 98] \\
\hline \multirow{2}{*}{$\begin{array}{l}\text { AGS } 3 \text { and } 4 \\
\text { without jets* }\end{array}$} & $78.9 \%$ & $100 \%$ & $100 \%$ & $86.2 \%$ & $90.9 \%$ \\
\hline & {$[60-97]$} & {$[76-100]$} & {$[76-100]$} & {$[68-96]$} & [83 - 99] \\
\hline
\end{tabular}


Figure 1

Comparison of SFU and Onen's hydronephrosis grading system AGS.

SFU grading system
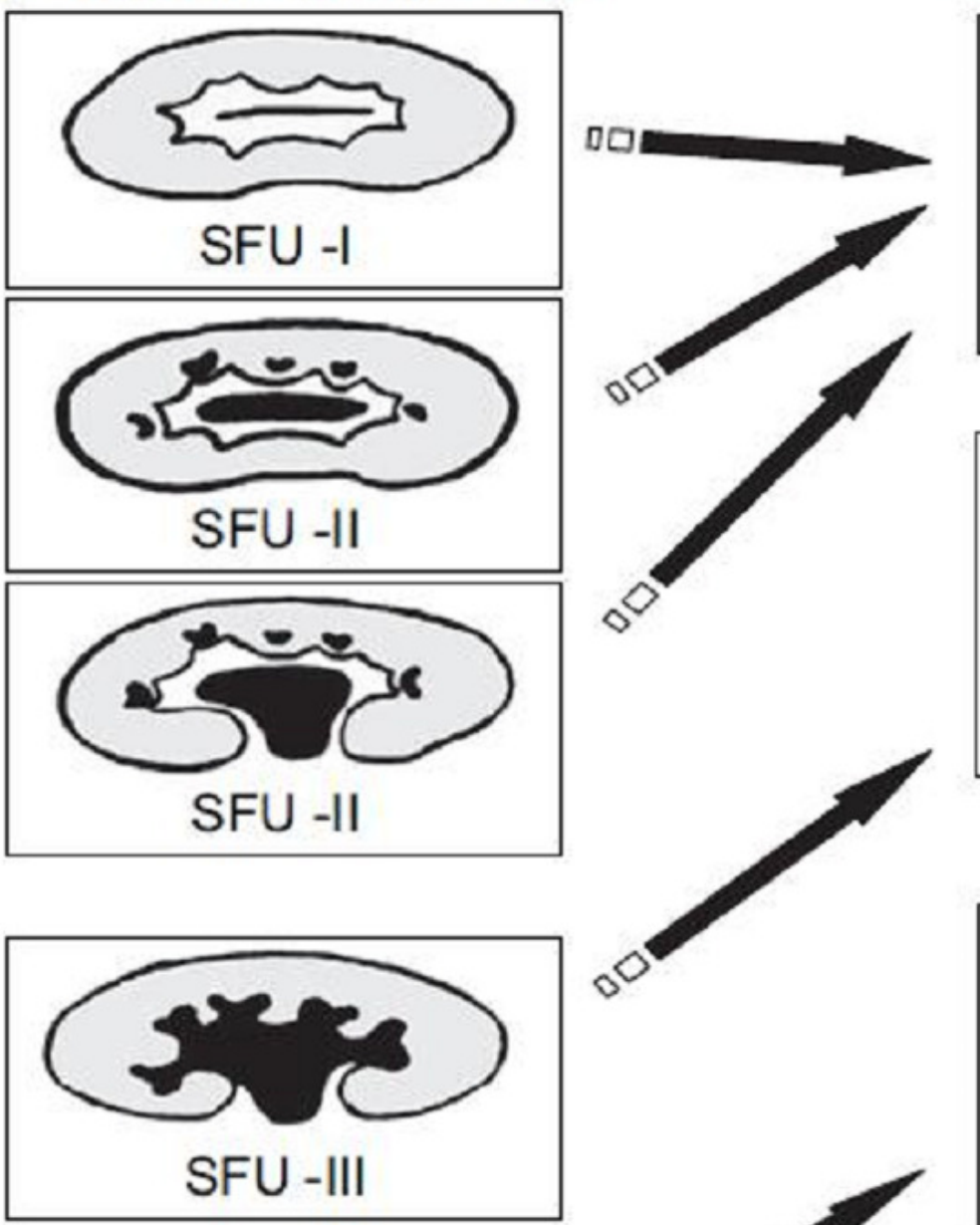

Onen's alternative grading
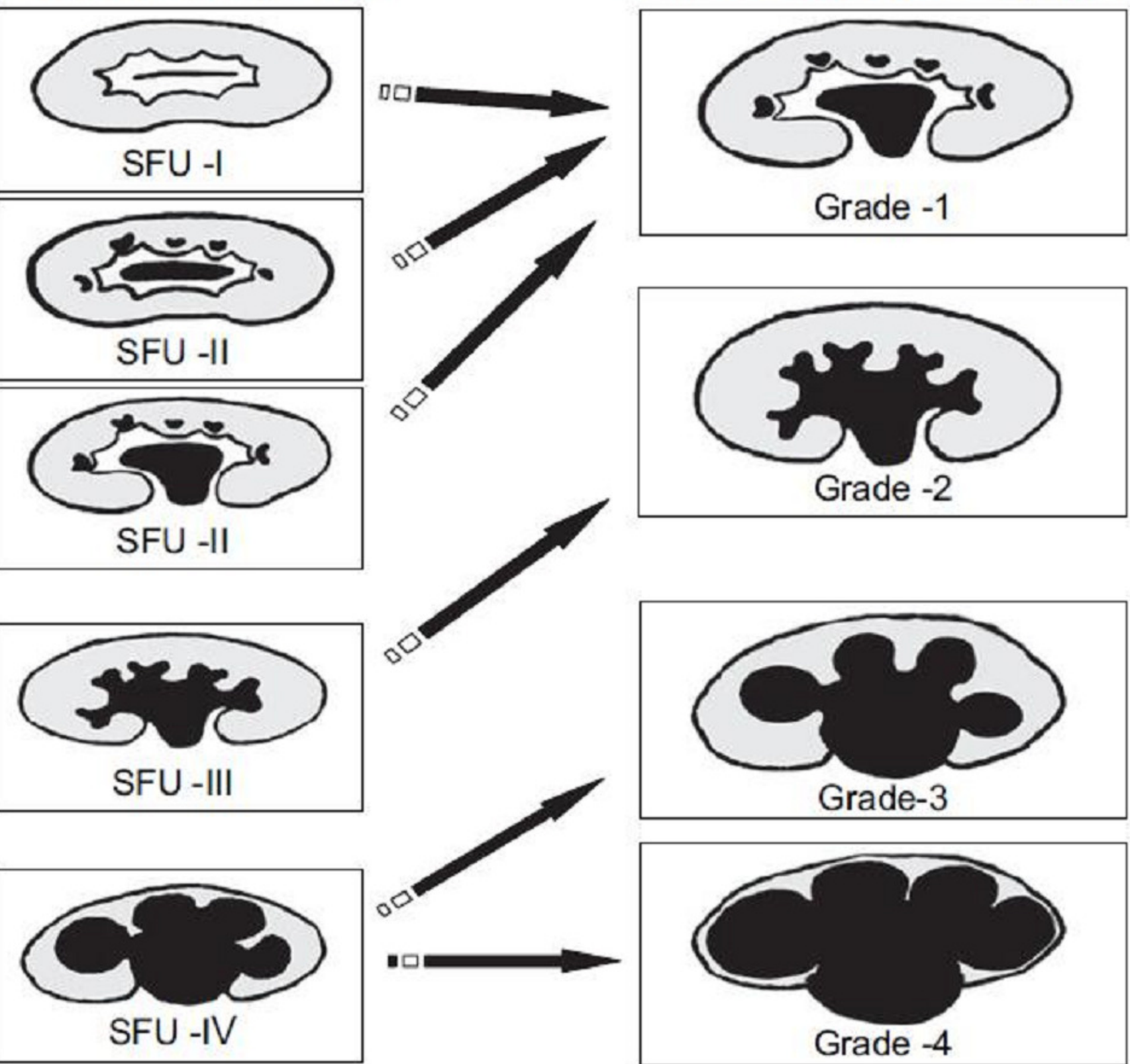
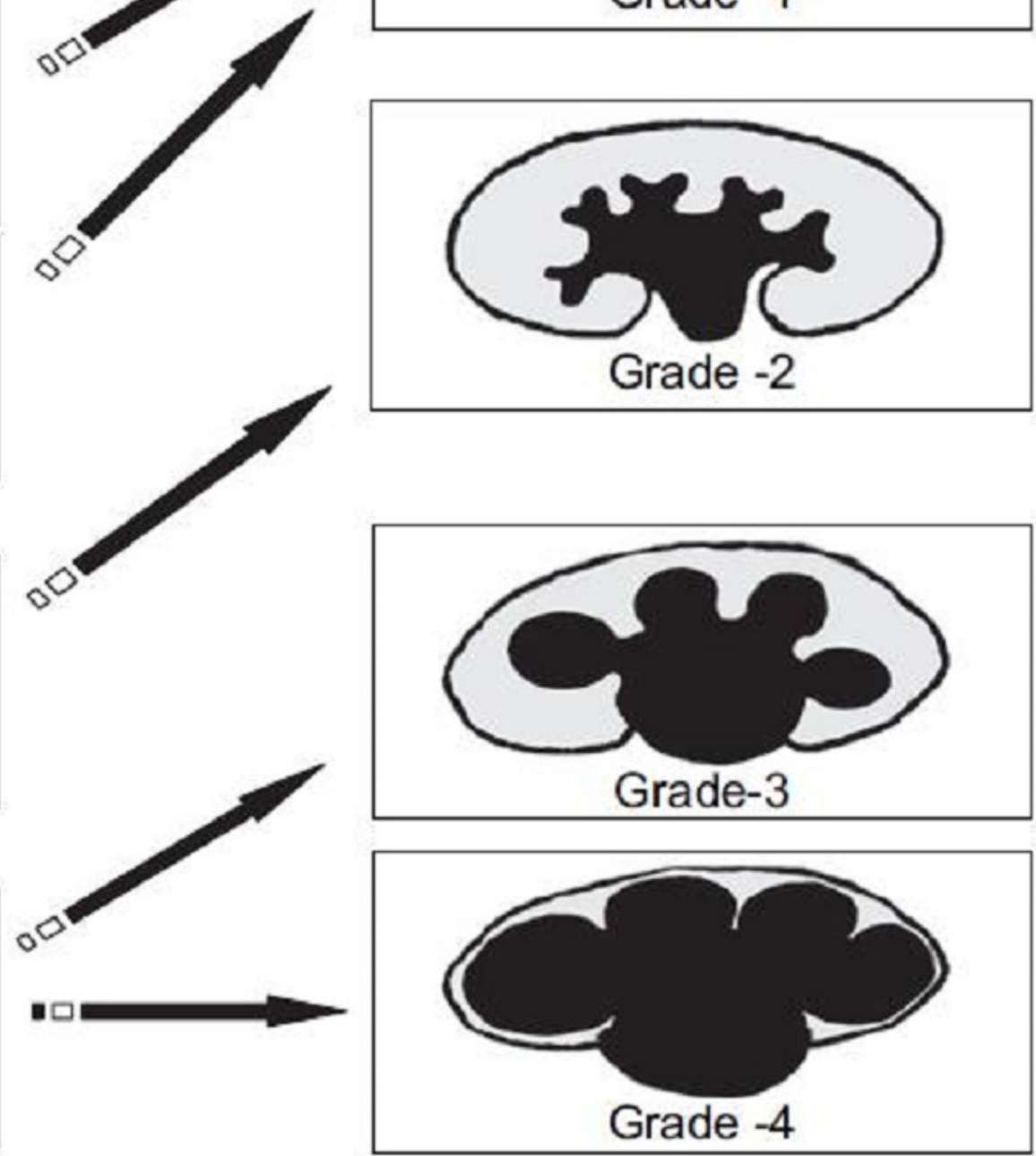
Figure 2

Flowchart of study design and test results.

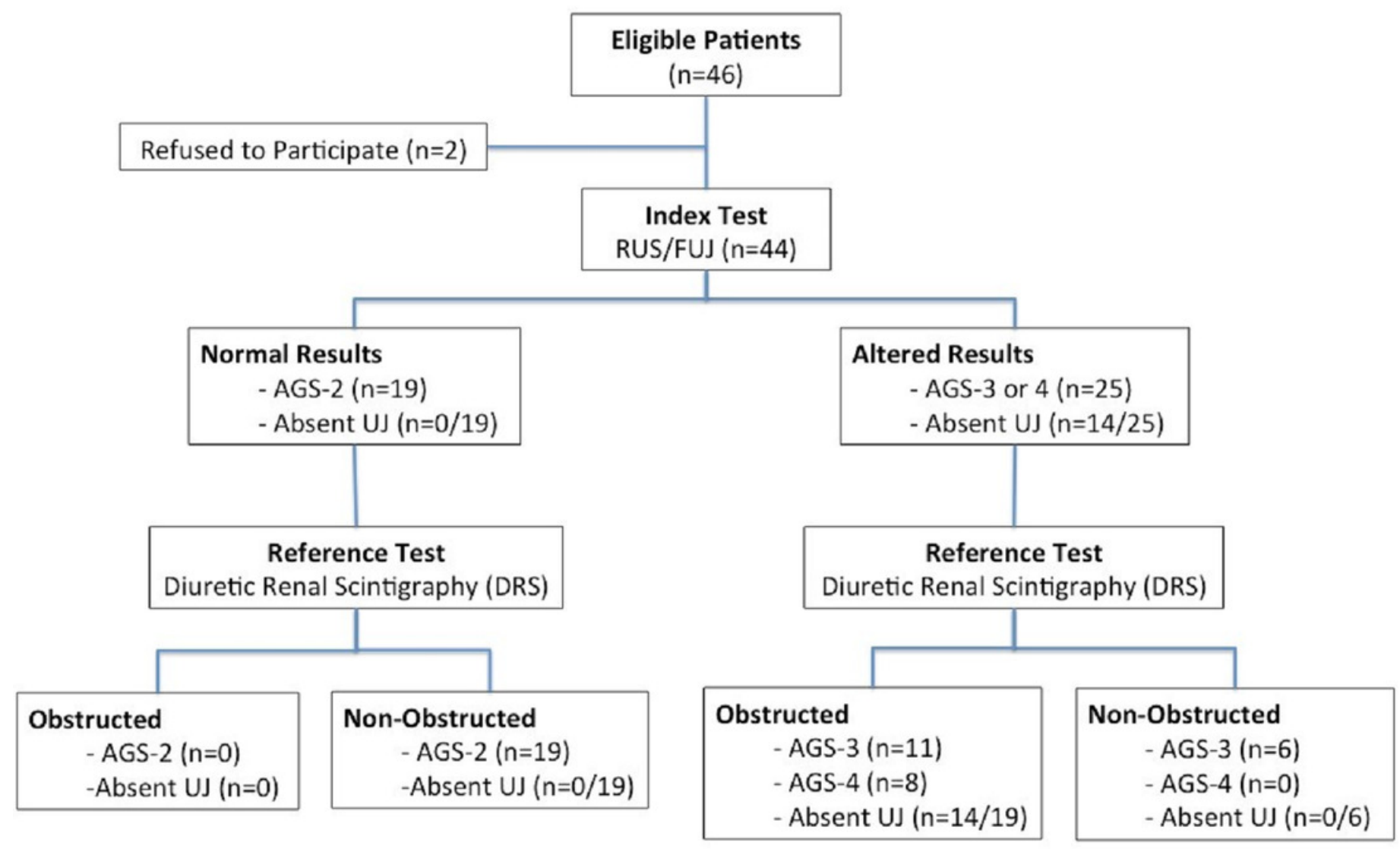


Figure 3

Differential renal function according to Onen's AGS.

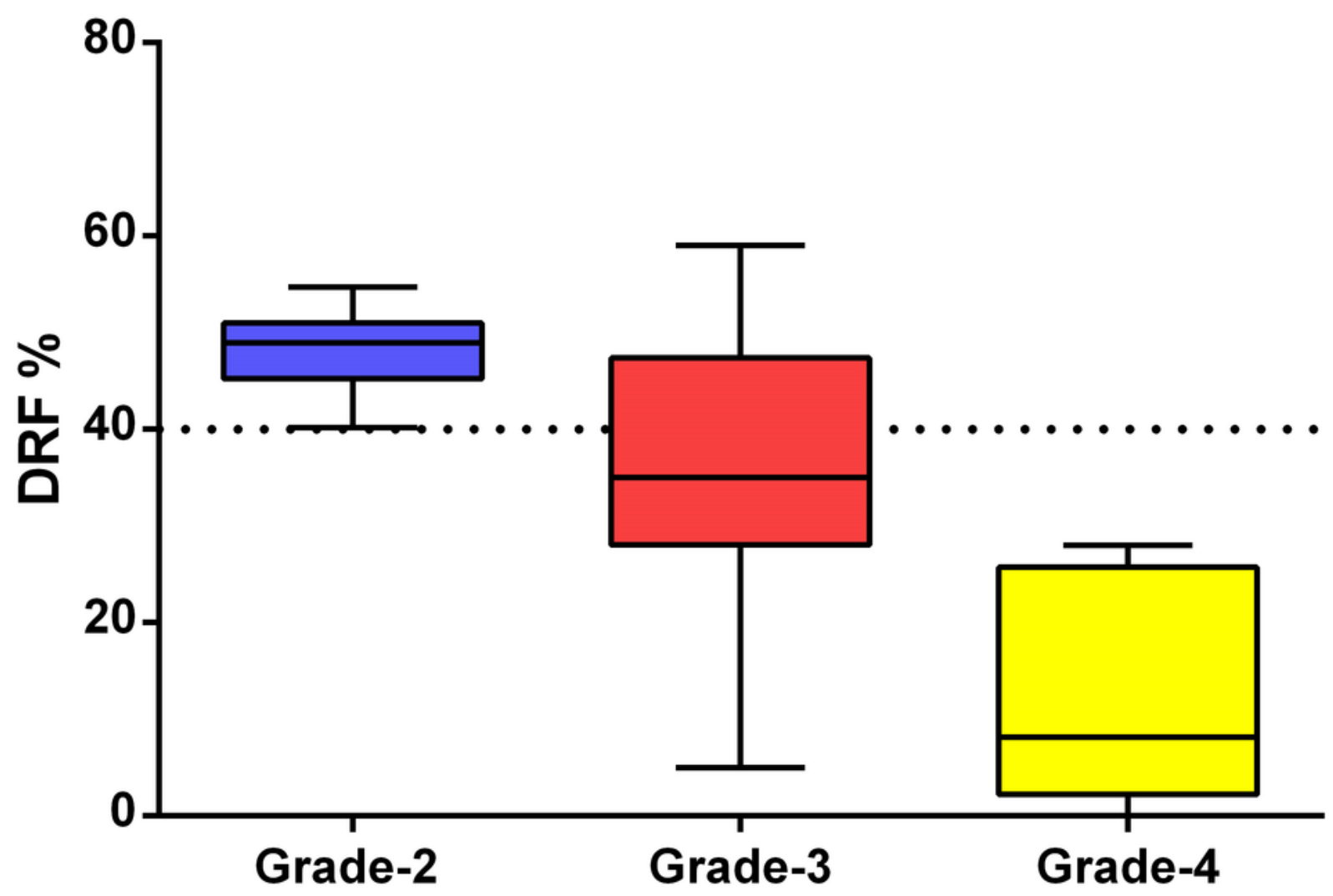

